\title{
H. H. PRICE, PARADIGMAS E O PROBLEMA DOS UNIVERSAIS
}

\author{
H. H. Price, Paradigms and the problem of universals
}

\author{
Valdetonio Pereira de Alencar \\ UFRJ/UFCA
}

\begin{abstract}
Resumo: Neste artigo, analiso o Nominalismo de Semelhança Aristocrático. O principal defensor dessa teoria foi H. H. Price. Inicialmente, apresento o Nominalismo de Semelhança Aristocrático. Price defende que o Realismo não funciona. Segundo ele, essa teoria não fornece uma correta explanação da semelhança inexata entre diferentes particulares. Além disso, ele analisa duas objeções clássicas contra o Nominalismo de Semelhança: o argumento do aspecto e o argumento do regresso. Posteriormente, analiso as principais críticas contra o Nominalismo de Price: o caráter primitivo da relação de semelhança, o problema dos paradigmas e o problema do regresso. A teoria de Price não é capaz de responder a essas objeções adequadamente.
\end{abstract}

Palavras-Chave: Price. Nominalismo de Semelhança. Paradigmas.

Abstract: In this paper, I discuss the Aristocratic Resemblance Nominalism. The main example of this theory is the one presented by H. H. Price. At first, I elucidate the Aristocratic Resemblance Nominalism. Price thinks Realism about universals does not work. According to him, this theory does not give an explanation of the similarity inexact between different individuals. Furthermore, he discusses two classical objections against Resemblance Nominalism: the respect argument and the regress argument. Later, I discuss three criticisms against the Price's Nominalism: resemblance is not primitive, the problem of paradigms and the regress problem. The Price's theory is not able to get rid of these objections.

Keywords: Price. Resemblance Nominalism. Paradigms.

Como todo Nominalismo, o Nominalismo de Semelhança rejeita a existência de universais enquanto entidades não redutíveis. O que o diferencia de outras formas de Nominalismo é a maneira como a nossa fala sobre propriedades é reduzida a uma fala sobre particulares. Para essa postura, o fato de um particular a possuir a propriedade $F$ é analisado como $a$ assemelhando-se aos F's. O fato de uma particular $a$ estar em uma relação $R$ com $b$ é analisado como o par ordenado $(a, b)$ assemelhando-se aos pares 
ordenados que satisfazem essa relação. O Nominalismo de Semelhança precisa a princípio postular particulares concretos e classes (que, normalmente, são consideradas particulares abstratos). Existe outra forma de Nominalismo que possui a mesma ontologia do Nominalismo de Semelhança: o Nominalismo de Classe. Existe, contudo, um aspecto que diferencia o Nominalismo de Semelhança do Nominalismo de Classe. Nesse último, a propriedade ser humano consiste na classe dos seres humanos. Assim como o realista, o nominalista de classe introduz uma entidade para explanar o que propriedades são. Isso não ocorre com a nominalista de semelhança ${ }^{1}$. Em geral, nessa teoria, não se define ontologicamente o que é uma propriedade. Antes, o fato de um objeto $a$ possuir a propriedade $F$ é que constitui o objeto da explanação. E nenhuma entidade, em especial, é introduzida para ocupar a função de $F$.

O Nominalismo de Semelhança é uma solução objetivista porque a semelhança entre diferentes particulares é um fato independente do representar humano ${ }^{2}$. Existem dois tipos de Nominalismo de Semelhança: o aristocrático e o igualitário. O Nominalismo de Semelhança Aristocrático foi formulado por Price. É denominado "aristocrático" devido à função atribuída aos paradigmas na formação de uma classe de semelhança. 0 fato de $a$ ser $F$ é analisado como $a$ assemelhando-se aos paradigmas definidores da classe dos F's. Rodriguez-Pereyra discorda de Price, defendendo uma forma igualitária de Nominalismo de Semelhança. O fato do objeto $a$ ser $F$ é analisado como $a$ assemelhando-se a todos os F's. A posição de Rodriguez-Pereyra pode ser chamada de "Nominalismo de Semelhança Igualitário", pois todos os objetos que possuem a propriedade $F$ possuem o mesmo status ${ }^{3}$.

Neste artigo, realiza-se uma análise do Nominalismo de Semelhança tal como formulado por H. H. Price. Na seção 1, apresento a posição de Price e suas críticas ao Realismo a respeito dos universais. Ele se dedica a refutar duas objeções clássicas: o argumento do aspecto e o argumento do regresso. Na seção 2, analiso as principais

\footnotetext{
${ }^{1}$ Apesar disso, o Nominalismo de Semelhança, em geral, ainda precisará de classes em sua ontologia para lidar com relações.

${ }^{2}$ Esse é um pressuposto do Nominalismo de Semelhança.

${ }^{3}$ RODRIGUEZ-PEREYRA, G. Resemblance nominalism: a solution to the problem of universals. Oxford: Oxford University Press, 2002.
} 
críticas contra o Nominalismo de Price: o caráter primitivo da relação de semelhança, o problema do estabelecimento de paradigmas e o problema do regresso.

\section{H. H. Price: críticas ao realismo e a defesa do nominalismo de semelhança}

Ao tratar do problema dos universais, Price coloca em oposição duas teorias: o Realismo aristotélico (in re) e o Nominalismo de Semelhança. O Realismo aristotélico é a tese de que universais devem estar presentes na constituição ontológica do mundo. Universais existem apenas instanciados nos particulares concretos (as substâncias primeiras de Aristóteles). Não existem, assim, universais não instanciados. 0 Nominalismo de semelhança tenta uma descrição da realidade pretensamente mais econômica, pois precisa apenas da categoria de particular para preencher a realidade. Apesar de divergirem em questões ontológicas, essas duas teorias, segundo Price, pretendem analisar os mesmos fatos objetivos. Price denomina esses fatos de "recorrência de características".

Intuitivamente, o mundo parece ser muito monótono, pois observamos uma incessante repetição: as mesmas cores, as mesmas formas, entre outras características, nos são apresentadas ao longo da nossa experiência. Apesar dessa forte intuição alicerçada no senso comum, filósofos, como Heráclito, tentaram negar isso. Pelo menos, é uma interpretação possível de seu aforismo: "tudo flui". Price discorda e utiliza um argumento baseado na forma como nós conhecemos o mundo: "In a world of incessant novelty, where there was no recurrence at all and no tedious repetitions, no concepts could ever be acquired; and thinking, even of the crudest and most primitive kind, could never begin"4.

Evidentemente, o filósofo heraclitiano poderia replicar que, de fato, todos os nossos conceitos são ilusórios e arbitrários, não captando a natureza processual da realidade. Além de ser uma conclusão implausível, talvez Price pudesse recorrer ao fato de que a utilização da linguagem já implica a aquisição de conceitos. Assim, mesmo a formulação heraclitiana "tudo flui" utiliza conceitos. Mas, se todo conceito é ilusório,

\footnotetext{
${ }^{4}$ PRICE, H. H. "What is the relationship between an individual and its characteristics" (1953). In: van INWAGEN, P. e ZIMMERMAN, D. W (Org.). Metaphysics: the big questions. Oxford, Blackwell, 1998, p. 23. 
temos que o filósofo heraclitiano dificilmente poderia formular a sua posição de forma coerente. Isso não implica que a posição Price esteja necessariamente correta, mas indica que talvez tenhamos que admitir a recorrência das características como único caminho na construção de uma solução para o problema dos universais.

Ao analisar o fenômeno da "recorrência de características", o Nominalismo de Semelhança e o Realismo Aristotélico divergem. Pode-se afirmar que a divergência encontra-se na análise da semelhança. O Realismo aristotélico entenderá que a relação de semelhança é derivativa da noção de universal. O objeto a assemelha-se ao objeto b porque $\mathbf{a}$ e $\mathbf{b}$ instanciam o mesmo universal. Um primeiro problema levantado contra 0 Realismo diz respeito ao fato da semelhança nem sempre ser exata. O caso das cores é um clássico da variação da semelhança em termos de intensidade. Podemos ter vários objetos brancos, mas que não são exatamente brancos. Para resolver esse problema, talvez seja necessário distinguir semelhança exata de semelhança inexata. Contudo, a ideia de que dois objetos assemelham-se porque instanciam o mesmo universal não parece deixar espaço para a noção de semelhança inexata. Isso aponta para o fato de que semelhança é primitiva e não derivada de um único universal presente em vários objetos. "Thus the philosophy of universals, when it makes all resemblance derivative, appears to forget that resemblances have degrees of intensity. Resemblance is treated as if it were degreeless, either present in its maximum degree or else not present at all" 5 . Além de variar em intensidade, semelhança também pode variar em extensão. A variação da semelhança em extensão está relacionada com a quantidade de propriedades que dois particulares compartilham. A princípio, Aristóteles é mais semelhante a Sócrates do que a um elefante porque compartilha com Sócrates um número maior de propriedades. Esse tipo de grau de semelhança não afeta o Realismo, podendo ser facilmente tratado a partir da noção de universal. Quanto mais universais um particular possui em comum com outro particular, mais eles se assemelham.

Depois de defender que semelhança é primitiva, Price analisa dois argumentos clássicos contra o Nominalismo de Semelhança. A sua análise dessas objeções é bastante

\footnotetext{
${ }^{5}$ PRICE, H. H. "What is the relationship between an individual and its characteristics" (1953). In: van INWAGEN, P. e ZIMMERMAN, D. W (Org.). Metaphysics: the big questions. Oxford, Blackwell, 1998, p. 30. 
interessante, pois ele introduz importantes elementos para a sua posição. O primeiro é o argumento do aspecto. Quando se afirma que a assemelha-se a $\mathbf{b}$, é provável que alguém pergunte: em qual aspecto? Objetos verdes assemelham-se em um aspecto, enquanto objetos redondos assemelham-se em outro. Analisar a classe dos objetos redondos recorrendo apenas ao fato de serem objetos que se assemelham entre si parece deixar a análise do Nominalista de Semelhança incompleta. Além disso, a necessidade de especificar qual a semelhança que os elementos de um conjunto de objetos apresentam entre si parece implicar a reintrodução de universais no Nominalismo de Semelhança. Essa objeção tenta mostrar que semelhança não é fundamental na constituição ontológica da realidade e que depende da noção de propriedade.

A fim de responder a essa objeção, Price introduz um elemento aristocrático em seu Nominalismo. Quando se pergunta a que os objetos redondos assemelham-se, não é necessário introduzir os aspectos. Segundo ele, objetos redondos, por exemplo, assemelham-se a certos objetos paradigmáticos. Toda classe tem um núcleo, alguns elementos padrões:

It is agreed by both parties that there is a class of red objects. The question is, what sort of structure does a class have? That is where the two philosophies differ. According to the Philosophy of Universals, a class is so to speak a promiscuous or equalitarian assemblage. All its members have, as it were, the same status in it. All of them are instances of the same universal, and no more can be said. But in the Philosophy of Resemblances a class has a more complex structure than this; not equalitarian, but aristocratic. Every class has, as it were, a nucleus, an inner ring of key members, consisting of a small group of standard objects or exemplars ${ }^{6}$.

Assim, objetos paradigmáticos forneceriam certa unidade a uma determinada classe natural. Para saber se um objeto pertence ou não a uma classe natural, deveríamos compará-lo com os objetos paradigmáticos da classe em questão.

Outra crítica clássica ao Nominalismo de Semelhança é o problema do regresso da semelhança. Segundo Russell, mesmo que o Nominalismo de Semelhança estivesse

\footnotetext{
${ }^{6}$ PRICE, H. H. "What is the relationship between an individual and its characteristics" (1953). In: van INWAGEN, P. e ZIMMERMAN, D. W (Org.). Metaphysics: the big questions. Oxford, Blackwell, 1998, p. 30. 
correto, essa posição comprometer-nos-ia com pelo menos um universal. Segundo Price, nenhuma razão é dada a favor da tese de que semelhança é um universal. Uma razão poderia ser dada recorrendo à Filosofia da Linguagem. "Semelhança" é uma palavra abstrata, assim como "azul", "ser humano" e "tigre". Nessa linha de raciocínio, a questão torna-se: palavras abstratas referem-se a universais? A argumentação diria que é impossível uma linguagem apenas com termos singulares sem palavras abstratas. Price não discorda dessa observação. Ele acredita que a mesma não possui nenhuma implicação ontológica, pois seria necessário demonstrar a seguinte premissa adicional: toda palavra abstrata denota um universal. O Nominalismo de Semelhança poderá explanar o significado da palavra "semelhança" através da observação de que existem semelhanças de várias ordens. Dois cachorros são semelhantes entre si em primeira ordem e dois objetos redondos são semelhantes entre si também em primeira ordem. A semelhança dos dois cachorros e a semelhança dos dois objetos redondos são semelhantes em segunda ordem. Existem, assim, semelhanças de primeira e de segunda ordem. Se o realista rejeitasse a noção de semelhança de segunda ordem, então seria possível utilizar a mesma crítica contra ele.

When it is said that 'cathood is a universal' the word 'universal' is itself a general word, just as 'cat' is when we say 'Pussy is a cat'. So according to the philosophy of universals, there must be a universal called 'universality'. And if it is a universal, universality must accordingly be an instance of itself. But this is a contradiction?

Uma boa solução para o realista, diz Price, seria diferenciar níveis da forma como ele mesmo fez para a relação de semelhança. Não é contraditório afirmar que um particular seria uma instância de uma universalidade de primeira ordem e esta seria uma instância da universalidade de segunda ordem. Nesse caso, o realista não mais poderia utilizar o argumento de Russell contra o Nominalismo de Semelhança.

Price faz ainda uma segunda crítica ao argumento de Russell. Semelhança não é apenas uma relação como as outras. Para o nominalista de semelhança, semelhança é algo muito fundamental para ser chamado de "relação". É fundamental porque, nessa

\footnotetext{
${ }^{7}$ Ibid., p. 35.
} 
espécie de Nominalismo, relações normalmente são analisadas através de semelhanças.

O autor considera, assim, que semelhança apresenta um caráter formal, devendo-se diferenciá-la de relações empíricas como "x está ao lado de y".

\section{Problemas com o nominalismo de Price}

Nesta seção, analiso várias críticas à teoria de Price. Sua posição foi duramente critica por Armstrong. Nesta seção, analisarei três problemas. Em primeiro lugar, discuto o caráter fundamental, ou não, da relação de semelhança. Em segundo lugar, discuto alguns problemas com a noção de paradigma. Por último, analiso se o Nominalismo de Price cai em algum dos regressos observados por Armstrong.

\subsection{Semelhança é primitiva ou derivada?}

A questão acima é central no debate sobre Nominalismo de Semelhança. É uma crítica clássica ao Nominalismo de Semelhança a de que a relação de semelhança não é fundamental, mas derivada da categoria de universal. Armstrong defende, de maneira intuitiva, o caráter derivativo da relação de semelhança: "It is natural to assert that things resemble because they have something in common, counter-intuitive to say that they have something in common because they resemble each other" ${ }^{\prime 2}$. Esse argumento não constitui uma refutação definitiva da tese de que semelhança é fundamental, pois recorre a uma mera intuição. Apesar de ser defensável o valor das intuições em argumentações filosóficas, o argumento de Armstrong, nesse caso, apenas atribui o ônus da prova de que semelhança é fundamental para o nominalista de semelhança. Assim, se esse não possuísse nenhum argumento correto de que semelhança é primitiva, então todo e qualquer Nominalismo de Semelhança seria facilmente refutado com a observação acima de Armstrong. Price, contudo, possui um argumento a favor do caráter primitivo da relação de semelhança. Segundo ele, semelhança nem sempre é exata no que diz respeito à intensidade. Você pode ter um conjunto de objetos que se assemelham apenas de maneira aproximada. Nesse caso, seria implausível afirmar que

\footnotetext{
${ }^{8}$ ARMSTRONG, D. M. A theory of universals: volume I: nominalism and realism. Cambridge: Cambridge University Press, 1978, p. 50.
} 
todos esses objetos instanciam o mesmo universal. Um exemplo clássico disso são as cores. Os elementos do conjunto dos objetos vermelhos assemelham-se apenas de forma aproximada. Alguns são mais vermelhos do que outros. Segundo o autor, a noção de universal não explana como a semelhança surge em graus. Isso aponta para o caráter primitivo da semelhança.

Price, contudo, reconhece haver uma objeção contra o argumento acima de que a relação de semelhança é primitiva. O realista poderia recorrer à distinção entre propriedades determinadas e propriedades determináveis para fornecer uma explanação da semelhança inexata. Essa distinção é relativa. A mesma propriedade pode ser determinada em relação a algumas propriedades e determinável em relação a outras propriedades. Essa distinção é elucidada adequadamente a partir de exemplos. A propriedade de ser mamífero é determinada em relação à propriedade ser vertebrado, que é uma propriedade determinável. As propriedades de ser primata e ser carnívoro são determinadas em relação à propriedade ser mamífero, que é uma propriedade determinável nesse contexto. Quando dois objetos não se assemelham em um nível máximo, eles compartilham a mesma propriedade determinável, mas não compartilham a mesma propriedade determinada. Assim, dois objetos vermelhos compartilham a mesma propriedade determinável ser vermelho, mas podem possuir diferentes propriedades determinadas em relação à propriedade determinável que eles compartilhem: um dos objetos pode ter a propriedade determinada ser vermelho escarlate e o outro objeto pode ter a propriedade determinada ser vermelho bordô. Price reconhece que o realista consegue, através da distinção acima, fornecer uma explanação da semelhança inexata, deixando o nominalista de semelhança sem argumento a favor do caráter primitivo da semelhança:

Inexact resemblance, we are invited to say, depends upon or is derived from the presence of the same determinable characteristic in a number of objects; exact resemblance (resemblance of maximum intensity) depends upon their being characterized by the same determinate characteristic ${ }^{9}$.

\footnotetext{
${ }^{9}$ PRICE, H. H. "What is the relationship between an individual and its characteristics" (1953). In: van INWAGEN, P. e ZIMMERMAN, D. W (Org.). Metaphysics: the big questions. Oxford, Blackwell, 1998, p. 38. 
Um segundo problema contra a posição de Price concerne aos exemplos utilizados por ele para sustentar a tese do caráter primitivo da relação de semelhança. Se você toma como exemplo propriedades físicas fundamentais, não vejo a existência de graus de semelhança em termos de intensidade. Tomem-se duas partículas atômicas com a propriedade ter carga elétrica. Enquanto uma tem carga elétrica positiva, a outra tem carga elétrica negativa. Não faz sentido afirmar que uma tem mais carga elétrica do que a outra. Muitos exemplos poderiam ser dados nos quais não teríamos grau de semelhança quanto à intensidade. Price fala da noção de graus de semelhança quanto à intensidade como se fosse algo corriqueiro. No entanto, ele só utiliza o exemplo das cores e o exemplo de formas geométricas. O exemplo das cores parece-me, em especial, muito problemático. Cores não são propriedades naturais. Uma cor é uma percepção visual. Propriedades geométricas também não parecem naturais. Da forma como ser circular é definido geometricamente, não parece deixar espaço para que um particular no mundo empírico seja circular.

Por conseguinte, sustento que os exemplos de Price não mostram uma gradação de semelhança em termos de intensidade. Apesar disso, ainda é possível falar de grau de semelhança em termos de extensão. Esse tipo de gradação, contudo, não mostra que semelhança é uma noção primitiva. O realismo pode analisar esse tipo de gradação através da noção de universal. Assim, Price não é bem sucedido em mostrar que semelhança é uma noção primitiva. Armstrong parece estar certo: as coisas são semelhantes porque possuem a mesma propriedade.

Por último, há outro problema com a defesa de Price do caráter fundamental da relação de semelhança. O Realismo ante rem platônico já reconhece graus de instanciação. O universal nesse Realismo é um modelo (uma forma). As diversas instâncias aproximam-se não exatamente desse modelo. Price admite que o Realismo ante rem platônico tenha sido a primeira posição a reconhecer graus de instanciação ${ }^{10}$. Nesse aspecto, a crítica dele dirige-se especialmente ao Realismo in re aristotélico. Infelizmente, ele não argumenta contra o Realismo ante rem. Ele sequer discute essa

\footnotetext{
${ }^{10}$ PRICE, H. H. "What is the relationship between an individual and its characteristics" (1953). In: van INWAGEN, P. e ZIMMERMAN, D. W (Org.). Metaphysics: the big questions. Oxford, Blackwell, 1998, p. 30. 
doutrina, porque, segundo Price, ela é mais distante do senso comum. Isso, claramente, não é um argumento. Assim, enquanto o Realismo aristotélico pode lidar facilmente com a variação de intensidade de uma propriedade através da distinção entre propriedades determinadas e propriedades determináveis, o Realismo platônico utiliza a noção de grau de instanciação. Price não é, pois, bem sucedido em explanar que a relação de semelhança não é derivável da noção de propriedade.

\title{
2.2 Problemas com os paradigmas
}

Vimos que a noção de objetos paradigmáticos foi introduzida por Price como resposta a uma das clássicas objeções contra o Nominalismo de Semelhança. Segundo essa objeção, o Nominalismo de Semelhança analisa o fato de que certos objetos são vermelhos, afirmando que eles se assemelham a outros objetos vermelhos. Contudo, essa análise parece incompleta, pois não especifica em qual aspecto esses objetos são semelhantes. Para especificar a semelhança entre os diversos objetos vermelhos, Price afirma que um objeto é vermelho porque se assemelha a certos objetos paradigmáticos.

Algumas críticas podem ser feitas contra a noção de objeto paradigmático. Armstrong observou que essa traz um certo grau de subjetividade para o Nominalismo de Semelhança:

\begin{abstract}
Resemblance Nominalism was earlier described as an Objectivist analysis. But it should be noted that the appeal to paradigms introduces a certain element of subjectivity, or at least of relativity. For it is clear that different people will normally have to use different paradigms for the same class of things. To that extent, for the Resemblance theorists, whiteness for you is not the same thing as whiteness for $\mathrm{me}^{11}$.
\end{abstract}

Como diz Armstrong, a noção de paradigma é, pelo menos, relativa, pois os paradigmas que alguém utiliza para definir a classe dos cachorros podem diferir dos paradigmas que outra pessoa utiliza para definir a mesma classe. Além disso, a noção de paradigma implica em certa subjetividade ${ }^{12}$ da noção de propriedade. Tome-se a

\footnotetext{
${ }^{11}$ ARMSTRONG, D. M. A theory of universals: volume I: nominalism and realism. Cambridge: Cambridge University Press, 1978, p. 46.

${ }^{12}$ Algo é subjetivo se e somente se depender do ser humano para ser ou existir.
} 
classe dos cachorros. A princípio, nenhum objeto dessa classe parece ter algo em si mesmo que o defina como paradigma. A noção de paradigma, contudo, parece fazer algum sentido se aceitarmos que existe grau de semelhança em termos de intensidade. Como alguns objetos parecem ser mais vermelhos do que outros, então talvez existam alguns objetos que objetivamente poderiam ser considerados paradigmáticos da classe dos objetos vermelhos. Aparentemente, exemplos como do das cores constituem exceções. Não parece ser possível estabelecer objetivamente o que constituiria um paradigma da classe dos seres humanos.

Um problema do caráter subjetivo dos paradigmas para Price diz respeito à coerência do seu Nominalismo. Segundo Price, Realismo e Nominalismo procuram fornecer uma análise de certos fatos objetivos que ele denomina "recorrência de características". É um fato objetivo que a é F. Esse fato é analisado afirmando-se que $a$ assemelha-se a paradigmas definidores da classe dos F's. Como paradigmas não são determinados de maneira objetiva, o Nominalismo de Price pode ser rotulado como uma solução subjetivista para o problema dos universais. E poderia, assim, sofrer as mesmas críticas dirigidas contra o Nominalismo de Predicado ${ }^{13}$ e o de Conceito ${ }^{14}$. Por isso, considero que a análise de Price é incompleta. Como, a princípio, determinamos, de maneira intersubjetiva, os paradigmas definidores de uma classe, paradigmas não constituem entidades objetivas.

Outro problema dos paradigmas concerne à possibilidade de propriedades unicamente instanciadas. É possível que apenas um objeto a possua uma propriedade $F^{15}$. Nesse caso, não haveria objetos paradigmáticos. À primeira vista, não poderíamos analisar o fato de que $a$ é $F$ recorrendo à semelhança com algum objeto paradigmático. Restaria ao nominalista de semelhança defender que $a$ é $F$ porque $a$ assemelha-se si mesmo. Por ser contraintuitivo, isso indica que semelhança deve ser analisada através de outra noção.

\footnotetext{
${ }^{13} \mathrm{O}$ nominalista de predicado defende que o fato do objeto $a$ possuir a propriedade $F$ deve ser analisado da seguinte maneira: o predicado " $\mathrm{F}$ " aplica-se a $a$.

${ }^{14} \mathrm{O}$ nominalista de conceito defende que o fato do objeto a possuir a propriedade $\mathrm{F}$ deve ser analisado da seguinte maneira: o conceito $\mathrm{F}$ aplica-se a $a$.

${ }^{15}$ ARMSTRONG, D. M. A theory of universals: volume I: nominalism and realism. Cambridge: Cambridge University Press, 1978, p. 50.
} 
Outro problema que os paradigmas trazem é o da sobredeterminação dos mesmos ${ }^{16}$. É possível que os mesmos paradigmas sirvam como paradigmas de classes distintas $^{17}$. Tomem-se dois fatos $a$ é $F$ e $b$ é G. Talvez a classe dos F's e a classe dos G's possuíssem os mesmos paradigmas. Nesse caso, o nominalista de semelhança aristocrático não teria como diferenciar a propriedade $F$ da propriedade $G$. Esse é um caso mais geral do problema da coextensionalidade. No caso das propriedades $P$ e $Q$ serem coextensivas, qualquer particular que tenha a propriedade $P$ e seja utilizado como paradigma também poderá ser um paradigma de $Q$. Essa teoria não possui elementos para bloquear essa possibilidade ${ }^{18}$.

\subsection{Semelhança é um universal?}

Armstrong diferenciou dois tipos de regresso que uma pretensa solução para o problema dos universais pode sofrer: o regresso do objeto e o regresso da relação. 0 objeto $a$ ter a propriedade $F$ é um fato analisado através da semelhança de $a$ com certos paradigmas. Como os paradigmas também possuem a propriedade $F$, então talvez faça sentido perguntar pela análise desse fato. Se o nominalista de semelhança tivesse que postular outros paradigmas para explanar o fato dos paradigmas originais serem $F$, então essa teoria está a caminho de cair no regresso do objeto.

Tome-se um paradigma $p$. O fato de que $p$ é $F$ pode ser tratado como se analisam outros particulares que são $F$. O paradigma $p$ possui a propriedade $F$ porque se assemelha aos paradigmas definidores da classe dos F's. Como entre esses paradigmas encontra-se o próprio $p$, faria parte da explanação de $p$ é $F$ que $p$ assemelha-se a $p$. Vimos que esse tipo de semelhança não parece muito intuitiva, sendo um problema para o nominalista de semelhança. Por isso, talvez uma solução mais interessante fosse considerar que o fato de que $p$ é $F$ fosse explanado recorrendo à semelhança de $p$ com os paradigmas da classe dos F's menos $p$. Nesse caso, o regresso parece ser bloqueado.

\footnotetext{
16 RAPHAEL, D. D. "Universals, Resemblances and Identity". Proceedings of the Aristotelian Society. London, v. 55, 1955, pp. 115-116.

${ }^{17}$ ARMSTRONG, D. M., op. cit., p. 48.

${ }^{18}$ ARMSTRONG, D. M. Universals: an opinionated introduction. San Francisco: Westview Press, 1989, p. 50.
} 
Qualquer paradigma $x$ de $F$ poderá ser explanado recorrendo ao mesmo artifício: $x$ é $F$ é analisado através da semelhança de $x$ com os paradigmas da classe dos F's menos $x$. Armstrong apresenta a seguinte restrição a essa solução:

This solution has the disadvantage that F-ness of a paradigm $F$ seems to become something different from the F-ness of a non-paradigm F. But perhaps this can be accepted. The standard metre is a metre long, but not quite in the same sense that other things are a metre long ${ }^{19}$.

O problema dessa solução é que dois particulares podem ter a mesma propriedade em virtude de estruturas distintas de semelhança. Um particular qualquer seria $F$ em virtude de assemelhar-se a certos paradigmas. Enquanto o paradigma $p$ seria F em virtude de assemelhar-se a um conjunto diferente de paradigmas. Armstrong não considera isso um problema. Ele recorre a uma analogia com o metro padrão. Se essa analogia for adequada, ela aponta para o caráter meramente convencional dos paradigmas. O metro padrão é um metro em um sentido meramente convencional, por isso é um metro em um sentido diferente das outras barras que possuem um metro. Um particular é um paradigma em virtude de uma escolha arbitrária. Apesar de a analogia ser adequada em mostrar como alguns conceitos são estabelecidos de maneira convencional, não parece adequada para tratar dos paradigmas de Price. O problema é analisar o fato do paradigma $p$ ter a propriedade $F$. Esse fato não é convencional. Ele é objetivo. O particular a possui a propriedade $F$ no mesmo sentido que $p$ tem a propriedade $F$. Assim, os fatos a é $F$ e $p$ é $F$ não devem ser analisados de formas diferentes por $p$ ser um paradigma. Isso parece impossibilitar uma correta análise de $p$ é $F$. Se analisarmos os fatos acima da mesma forma, retornaremos ao problema anterior. Faria parte da explanação de $p$ é $F$ que $p$ assemelha-se a si mesmo, o que não parece uma análise adequada.

Outro tipo de regresso que é considerado mais sério para o Nominalismo de Semelhança é o regresso da relação. No caso dessa forma de Nominalismo, a relação em questão é a de semelhança. Apesar de Price afirmar que nenhum argumento é dado

\footnotetext{
${ }^{19}$ ARMSTRONG, D. M. "Infinite Regress Arguments and the Problem of Universals". Australasian Journal of Philosophy. Sydney, 52, 1974, p. 195.
} 
a favor da tese de que semelhança é um universal, Russell apresenta um interessante argumento:

\begin{abstract}
If we wish to avoid the universals whiteness and triangularity, we shall choose some particular patch of white or some particular triangle, and say that anything is white or a triangle if it has the right sort of resemblance to our chosen particular. But then the resemblance required will have to be a universal. Since there are many white things, the resemblance must hold between many pairs of particular white things; and this is the characteristic of a universal. It will be useless to say that there is a different resemblance for each pair, for then we shall have to say that these resemblances resemble each other, and thus at last we shall be forced to admit resemblance as a universal. The relation of resemblance, therefore, must be a true universal. And having been forced to admit this universal, we find that it is no longer worth while to invent difficult and unplausible theories to avoid the admission of such universals as whiteness and triangularity ${ }^{20}$.
\end{abstract}

O argumento de Russell consiste em mostrar que semelhança possui características que universais normalmente possuem. Uma característica típica da noção de universal consiste em ser multiplamente instanciada. Semelhança pode ser encontrada em diferentes pares de particulares. Como semelhança apresenta um caráter repetível, então semelhança seria um universal. Uma réplica do nominalista seria afirmar que semelhança não é um universal porque os pares de particulares não se assemelham de forma exata. Nesse caso, a semelhança de um determinado par seria particular. Isso geraria um regresso, pois as diferentes semelhanças seriam semelhantes. Temos, assim, uma semelhança de segunda ordem. Se essa semelhança também for um particular, então teremos uma semelhança de terceira ordem, e assim por diante. Esse regresso não é muito interessante para o nominalista porque o regresso não é ontologicamente econômico. Mesmo que as semelhanças sejam particulares, é introduzido um novo tipo de entidade, que são os particulares abstratos (tropos).

Cargile $^{21}$ sustenta que se pode bloquear o problema o regresso utilizando os paradigmas. A fim de realizar esse objetivo, ele pressupõe duas teses: (i) O mundo é composto por um número finito de objetos e (ii) Devemos levar em consideração apenas

\footnotetext{
${ }^{20}$ RUSSELL, Bertrand. The problems of philosophy (1912). La Vergne: Watchmaker Publishing, 2009, pp. 70-71.

${ }^{21}$ CARGILE, James. "On Russell's Argument against Resemblance Nominalism". Australasian Journal of Philosophy. Sydney, 81, 2003, pp. 555-557.
} 
as semelhanças entre particulares não paradigmáticos e os paradigmáticos. Suponha haver três objetos brancos: $a, b$ e $c$. Tome-se $a$ o objeto paradigmaticamente branco nesse caso. Temos, pois, duas semelhanças: s1 (a semelhança entra $a$ e $c$ ) e s2 (a semelhança entre $b$ e $c$ ). O nominalista de semelhança aristocrático não precisa admitir a semelhança entre $a$ e $b$. Essa semelhança não tem nenhuma função nessa forma de Nominalismo. Não serve para explanar que os objetos $a, b$ e $c$ são brancos. Contudo, há uma semelhança de ordem 2: a semelhança entre s1 e s2. Como há apenas essa semelhança de segunda ordem, não é possível formular, nesse caso, uma semelhança de terceira ordem. O regresso é bloqueado. Não importa a quantidade de objetos brancos, desde que seja um número finito. Sempre teremos uma quantidade finita de semelhanças e, por conseguinte, o regresso sempre é bloqueado.

A estratégia de Cargile foi criticada em alguns aspectos. Rodriguez-Pereyra ${ }^{22}$ considera que a tese (i) não é metafisicamente neutra. Caso seja necessário admitir o Realismo Modal sobre mundos possíveis, então teríamos uma quantidade infinita de objetos brancos ao longo dos mundos possíveis. Um segundo problema, levantado por Rodriguez-Pereyra ${ }^{23}$, questiona se a estratégia de Cargile é eficaz em bloquear o regresso. Como os objetos $b$ e $c$ são semelhantes, teríamos que admitir s3 (a semelhança entre $b$ e $c$ ). Ora, não parece relevante se essa semelhança é utilizada para explanar o fato de $b$, por exemplo, possuir uma propriedade. É fato que esses objetos são semelhantes. Assim, teríamos três semelhanças de primeira ordem: s1, s2 e s3. Temos que: s1 é semelhante a s2, s2 é semelhante a s3 e s1 é semelhante a s3. Teríamos, assim, três semelhanças de segunda ordem: S1, S2 e S3. Como essas entidades são semelhantes entre si, haveria três semelhanças de terceira ordem. O regresso ao infinito não poderia ser bloqueado.

Mesmo que a estratégia de Cargile funcionasse para bloquear o recesso, o nominalista de semelhança ainda teria que admitir que semelhanças entre os

22 RODRIGUEZ-PEREYRA, G. "Paradigms and Russell's Resemblance Regress". Australasian Journal of Philosophy. Sydney, 82 (4), 2004, p. 647.

${ }^{23}$ Ibid., p. 648. 
particulares $a, b$ e $c$ são entidades. Rodriguez-Pereyra ${ }^{24}$ considera que s1 e s2 devam ser particulares, pois o nominalista de semelhança não quer admitir universais em sua ontologia. Contudo, s1 e s2 não pertencem à mesma categoria ontológica que $a$ e $b$. Essas últimas pertencem à categoria ontológica de particular concreto. Uma entidade como a semelhança entre $a$ e $b$ deve ser categorizada como um tropo, isto é, como uma propriedade particularizada. Nesse caso, o nominalista de semelhança, a fim de bloquear o regresso, teria que admitir mais um tipo de entidade em sua ontologia.

Um nominalista de semelhança, como Price, poderia argumentar que o regresso da relação é um problema presente em toda solução relacional do problema dos universais. O Realismo, por exemplo, também introduz uma relação para explanar a recorrência das propriedades, que é a relação de instanciação. Armstrong defendeu que toda solução para o problema dos universais apresenta esse problema ${ }^{25}$.

\section{Conclusão}

O Nominalismo desenvolvido por Price utiliza a mesma noção de propriedade do Realismo. Propriedades naturais são objetivas. A solução dele consiste em utilizar a relação de semelhança e a noção de paradigma para analisar o fato de um particular ter uma determinada propriedade. O problema aparece porque nem a relação de semelhança, nem a noção de paradigma conseguem fornecer uma análise completa das propriedades naturais. A noção de paradigma falha nesse quesito devido a seu caráter não objetivo. Por isso, não podemos reduzir a nossa fala sobre propriedades a uma fala sobre paradigmas. Pensando o Nominalismo de Semelhança de uma forma geral, o problema dos paradigmas parece ser menos destrutivo, pois existe, pelo menos, uma versão desse tipo de Nominalismo, desenvolvida por Rodriguez-Pereyra, que não utiliza paradigmas.

Quanto à relação de semelhança, Price não consegue mostrar que ela é fundamental. Particulares são semelhantes entre si, mas isso parece ser o caso porque

\footnotetext{
${ }^{24}$ RODRIGUEZ-PEREYRA, G. "Paradigms and Russell's Resemblance Regress". Australasian Journal of Philosophy. Sydney, 82 (4), 2004, p. 651.

${ }^{25}$ ARMSTRONG, D. M. "Infinite Regress Arguments and the Problem of Universals". Australasian Journal of Philosophy. Sydney, 52, 1974, p. 198.
} 
eles possuem algo em comum, o que torna essa semelhança algo meramente superveniente às propriedades. O problema do caráter não derivado da relação de semelhança é uma questão mais desafiadora para qualquer forma de Nominalismo de Semelhança. Não bastaria a suposta da economia ontológica que essa posição aparentemente apresenta. Mesmo que não possuísse nenhum problema, ela ainda teria que demonstrar que o fato de $a$ ser $F$ ocorre porque $a$ é semelhante a outros Fs. É mais intuitivo pensar que $a$ é semelhante a outros $F s$ porque $a$ é $F$.

\section{Referências bibliográficas}

ARMSTRONG, D. M. "Infinite Regress Arguments and the Problem of Universals". Australasian Journal of Philosophy. Sydney, 52, 1974, pp.191-201.

A theory of universals: volume I: nominalism and realism. Cambridge: Cambridge University Press, 1978.

. Universals: an opinionated introduction. San Francisco: Westview Press, 1989.

CARGILE, James. "On Russell's Argument against Resemblance Nominalism". Australasian Journal of Philosophy. Sydney, 81, 2003, pp. 549-560.

PRICE, H. H. "What is the relationship between an individual and its characteristics" (1953). In: van INWAGEN, P. e ZIMMERMAN, D. W (Org.). Metaphysics: the big questions. Oxford, Blackwell, 1998, pp. 23-40.

RAPHAEL, D. D. "Universals, Resemblances and Identity". Proceedings of the Aristotelian Society. London, v. 55, 1955, pp. 109-132.

RODRIGUEZ-PEREYRA, G. Resemblance nominalism: a solution to the problem of universals. Oxford: Oxford University Press, 2002.

. "Paradigms and Russell's Resemblance Regress". Australasian Journal of Philosophy. Sydney, 82 (4), 2004, pp. 644-51.

RUSSELL, Bertrand. The problems of philosophy (1912). La Vergne: Watchmaker Publishing, 2009. 